Virginia Commonwealth University VCU Scholars Compass

2018

\title{
Curvature dependence of the effect of ionic functionalization on the attraction among nanoparticles in dispersion
}

B. Shadrack Jabes

Virginia Commonwealth University

D. Bratko

Virginia Commonwealth University, dbratko@vcu.edu

Alenka Luzar

Virginia Commonwealth University

Follow this and additional works at: https://scholarscompass.vcu.edu/chem_pubs

Part of the Chemistry Commons

(C) 2018 Author(s).

\section{Downloaded from}

https://scholarscompass.vcu.edu/chem_pubs/96

This Article is brought to you for free and open access by the Dept. of Chemistry at VCU Scholars Compass. It has been accepted for inclusion in Chemistry Publications by an authorized administrator of VCU Scholars Compass. For more information, please contact libcompass@vcu.edu. 


\section{Curvature dependence of the effect of ionic functionalization on the attraction among nanoparticles in dispersion}

Cite as: J. Chem. Phys. 148, 222815 (2018); https://doi.org/10.1063/1.5017525

Submitted: 27 November 2017 . Accepted: 13 February 2018 . Published Online: 16 March 2018

B. Shadrack Jabes, Dusan Bratko (D), and Alenka Luzar (i)

\section{COLLECTIONS}

Paper published as part of the special topic on lons in Water
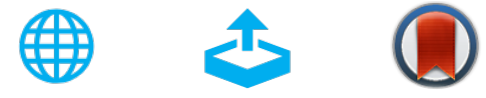

\section{ARTICLES YOU MAY BE INTERESTED IN}

A simulation study of homogeneous ice nucleation in supercooled salty water

The Journal of Chemical Physics 148, 222811 (2018); https://doi.org/10.1063/1.5008889

The influence of polarizability and charge transfer on specific ion effects in the dynamics of aqueous salt solutions

The Journal of Chemical Physics 148, 222803 (2018); https://doi.org/10.1063/1.5012682

2D nanoporous membrane for cation removal from water: Effects of ionic valence, membrane hydrophobicity, and pore size

The Journal of Chemical Physics 148, 222804 (2018); https://doi.org/10.1063/1.5013926

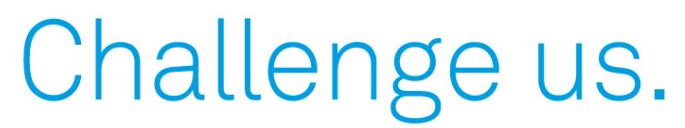

What are your needs for periodic signal detection?

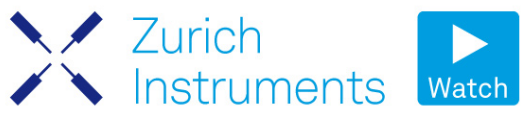

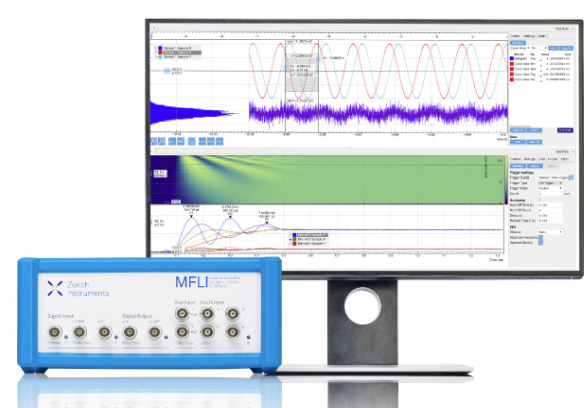

148, 222815 


\title{
Curvature dependence of the effect of ionic functionalization on the attraction among nanoparticles in dispersion
}

\author{
B. Shadrack Jabes, Dusan Bratko, ${ }^{\text {a) }}$ and Alenka Luzarb) \\ Department of Chemistry, Virginia Commonwealth University, Richmond, Virginia 23284, USA
}

(Received 27 November 2017; accepted 13 February 2018; published online 16 March 2018)

\begin{abstract}
Solubilization of nanoparticles facilitates nanomaterial processing and enables new applications. An effective method to improve dispersibility in water is provided by ionic functionalization. We explore how the necessary extent of functionalization depends on the particle geometry. Using molecular dynamics/umbrella sampling simulations, we determine the effect of the solute curvature on solventaveraged interactions among ionizing graphitic nanoparticles in aqueous dispersion. We tune the hydrophilicity of molecular-brush coated fullerenes, carbon nanotubes, and graphane platelets by gradually replacing a fraction of the methyl end groups of the alkyl coating by the ionizing -COOK or $-\mathrm{NH}_{3} \mathrm{Cl}$ groups. To assess the change in nanoparticles' dispersibility in water, we determine the potential-of-mean-force profiles at varied degrees of ionization. When the coating comprises only propyl groups, the attraction between the hydrophobic particles intensifies from spherical to cylindrical to planar geometry. This is explained by the increasing fraction of surface groups that can be brought into contact and the reduced access to water molecules, both following the above sequence. When ionic groups are added, however, the dispersibility increases in the opposite order, with the biggest effect in the planar geometry and the smallest in the spherical geometry. These results highlight the important role of geometry in nanoparticle solubilization by ionic functionalities, with about twice higher threshold surface charge necessary to stabilize a dispersion of spherical than planar particles. At $25 \%-50 \%$ ionization, the potential of mean force reaches a plateau because of the counterion condensation and saturated brush hydration. Moreover, the increase in the fraction of ionic groups can weaken the repulsion through counterion correlations between adjacent nanoparticles. High degrees of ionization and concomitant ionic screening gradually reduce the differences among surface interactions in distinct geometries until an essentially curvature-independent dispersion environment is created. Insights into tuning nanoparticle interactions can guide the synthesis of a broad class of nonpolar nanoparticles, where solubility is achieved by ionic functionalization. Published by AIP Publishing. https://doi.org/10.1063/1.5017525
\end{abstract}

\section{INTRODUCTION}

Graphitic nanoparticles such as fullerenes, carbon nanotubes (CNTs), and graphene and its derivatives ${ }^{1}$ like graphane $^{2,3}$ represent an interesting new class of synthetic carbon-based nanomaterials (CBNs). The richness of structural features of CBNs derives from the presence of pentagon and heptagon units within the network of hexagonal lattice that give rise to the positive and negative curvatures and varied dimensionalities. ${ }^{4,5}$ The physicochemical features of CBNs include strong van der Waals attractions among the particles ${ }^{6}$ that hamper their dispersing ability and solubility in both aqueous ${ }^{7-10}$ and organic ${ }^{7,11,12}$ solvents. From the material science perspective, this poses limitations in fabricating nanocomposites, combining the unique properties of multiple nanomaterials homogeneously, and in the processing of engineered CBNs in applications including electronics, optoelectronics, photovoltaic devices, and chemical and biological sensors. ${ }^{13}$ On the other hand, CBNs can be internalized

a)dbratko@vcu.edu

b)aluzar@vcu.edu within the cellular environment. ${ }^{14-16}$ Their unique electrical, thermal, and spectroscopic properties facilitate CBNs' detection and monitoring of their function in therapeutic applications. However, the lack of biodegradability and solubility in aqueous media reduce their biocompatibility, imposing limitations in bio-medical applications like bioimaging ${ }^{17}$ and targeted delivery of biomacromolecules into the cellular environment. ${ }^{18-20}$ Understanding and controlling the solubility properties of graphitic nanoparticles, such as the fragments of graphene, carbon nanotubes (CNTs), and their derivatives, holds promise for exciting new applications. Surface modifications proposed to disperse CBNs in aqueous media include chemical functionalization by polar and ionic groups. $^{21-27}$

We are interested in chemical modifications toward increasing the hydrophilicity of carbon-based nanoparticles by planting ionic functional groups such as ethyl- $\mathrm{COO}^{-}$or ethyl- $\mathrm{NH}_{3}^{+}$which not only bring up new biological properties but can also assist energy technologies. ${ }^{28}$ For example, experimental studies suggest that carboxylic derivatives of $\mathrm{C}_{60}$ functionalized with malonic acid fragments possess excellent biological properties (antibacterial activity) ${ }^{29}$ and have 
been observed to prevent bacteria induced meningitis. ${ }^{30} \mathrm{On}$ the other hand, carboxylate and amine functionalized multiwall CNTs dispersed in aqueous solution can be assembled into thin porous films by using a layer-by-layer technique. Such films can be used to design electrode materials for fuel cells, where chemical energy from fuel can be converted into electricity. ${ }^{28,31}$

Experimental insights have been complemented by molecular dynamics simulations of hydration, mechanical properties, and solution interactions between chemically modified CBN particles including charge-bearing $\mathrm{C}_{60}{ }^{32}$ or CNTs, ${ }^{33}$ graphene fragments ${ }^{34-37}$ as well as CNTs, and extended planar surfaces with polar ${ }^{38-40}$ or ionic ${ }^{41}$ functionalities. However, the general guidelines toward the design of functionalized CBN particles with targeted wetting or solubility characteristics are still lacking. In recent simulation studies, we have shown $^{39,41}$ that a variety of chemical modifications are possible to tune the hydrophilicity without altering the thickness or density of the surface coating, just by replacing a fraction of alkyl surface groups by polar ${ }^{39}$ or ionic ${ }^{41}$ ones. We also showed how the hydrophobicity of homogeneous nanoparticles depends on their geometry. ${ }^{42}$ For alkyl-covered surfaces, the apparent hydrophobicity, quantified in terms of interfacial compressibility ${ }^{43,44}$ of water, increased upon the transition from spherical to cylindrical to planar particle geometry. ${ }^{42}$ The change was consistent with simultaneous strengthening of the hydrophobic attraction between the particles.

In the present work, we examine the role of particle geometry on the effectiveness of ionic functionalization in reducing the dispersed particles' propensity for association. To this end, we densely coat the solutes with propyl chains $\left(-\mathrm{CH}_{2}-\mathrm{CH}_{2}-\mathrm{CH}_{3}\right)$ and then tune the surface hydrophilicity by substituting a fraction of terminal methyl groups with ionizing $\left(-\mathrm{NH}_{3}^{+} \mathrm{Cl}^{-}\right.$or $\left.-\mathrm{COO}^{-} \mathrm{K}^{+}\right)$ones. In a system with planar geometry such as the functionalized graphane, the relation between the surface free energy, the density of functional groups that control the hydrophobic nature of the solute, and their distribution are well established. ${ }^{41,45}$ The average density of charges required to turn the solvent-induced force between alkyl-covered plates repulsive was found to be close to 0.08 elementary charges per $\mathrm{nm}^{2}$. This density corresponded to about $2 \%$ of alkyl groups replaced by the ionic ones. ${ }^{41}$ But what percentage of ionic substitutions would suffice to stabilize the dispersion of carbon nanoparticles or solubilize carbon nanoparticles with different curvatures in water? The poor solubility of carbon structures is attributed to strong attractive dispersion forces among the particles rather than to the lack of affinity to water. ${ }^{46-48}$ This attraction can be overcome by introducing surface charges ${ }^{32}$ through adsorption, or by experimentally feasible ionic functionalization, but the density of charges must be increased substantially. The question we wish to answer is, how does the threshold charge density of the attraction-to-repulsion transition depend on the particle geometry? In the absence of screening ions, the electric field strength around a planar, cylindrical, or spherical charged particle varies with the distance $r$ approximately as $r^{0}, r^{-1}$, or $r^{-2}$, respectively. It is therefore clear that charge functionalization will be most effective in the planar geometry and least in the spherical geometry. We compare the three geometries of carbon-based nanomaterials by focusing on the potential of mean force $W(r)$ among adjacent particles and its crossover from attraction to repulsion upon increasing the surface charge. While the thermodynamic solubility also depends on the energetics of the precipitated phase, the repulsive potential of mean force in solution indicates a stable or, at the very least, a metastable dispersion state incapable of particle association and nucleation of the solid phase. We find that the chargeinduced repulsion reaches the maximal strength at optimal shape-specific surface densities of ionic groups. To explain the nonmonotonic effects of the surface charge and the observed differences between the interparticle forces in the three geometries, we also monitor the distribution of counterions and water molecules around the solute. We pay particular attention to the coordination number of the counterions as a measure of ion condensation, which determines the optimal extents of ionic functionalization in each of the distinct particle geometries we consider.

\section{MODELS}

In this section, we briefly describe the models we used in simulating the solvent-averaged interaction among ionizing nanoparticles in water. To span the broad range from strongly hydrophobic to strongly hydrophilic particles of invariable shapes, we use propyl-coated carbon nanoparticles ${ }^{42}$ with additional ionic substitutions. We consider three principal particle geometries: planar (finite size fragments of graphane, ${ }^{2,3}$ a hydrogen-saturated form of graphene), cylindrical (carbon nanotubes), and spherical (fullerene $\mathrm{C}_{60}$ ) (see Fig. 1 left). We choose the fully saturated $\left(\mathrm{C}_{n} \mathrm{H}_{n}\right)$ graphane structure to allow functionalization without inducing structural deformations upon the changes of carbon hybridization. In all three geometries, the areal density of grafted propyl chains corresponds to $\sim 4$ terminal methyl groups per $\mathrm{nm}^{2}$ of the solvent-exposed particle surface, similar to the densities of self-assembled monolayers. ${ }^{49,50}$ Graphane plates are functionalized only on one side. For fullerene $\left(\mathrm{C}_{60}\right)$, we obtain the target density by a uniform grafting of 24 propyl groups, yielding an approximately spherical particle with the excluded volume diameter $\sigma \sim 17.3 \AA$. For the definition of $\sigma$, see the caption of Fig. 1. We choose the CNTs with a diameter identical to that of the fullerene, and the platelets are assigned the width of $\sim 16.5 \AA$. The length of the platelets and CNTs is $\sim 2.5$ times the diameter of the functionalized fullerene or CNT, leading to the sizes of 366 or 255 backbone carbon atoms and 60 or 19 grafted propyl groups on the CNT or the graphane platelet backbones, respectively. The numbers of propyl chains are not proportional to the number of backbone carbon atoms because, with curved particles (fullerenes and CNTs), the density of planted groups at the backbone exceeds the predetermined areal density at the plane of contact with the solvent.

In order to achieve the desired percentage of ionic functionalization, the end groups of alkyl chains were gradually replaced by the $-\mathrm{NH}_{3}^{+} \mathrm{Cl}^{-}$or $-\mathrm{COO}^{-} \mathrm{K}^{+}$groups. Approximately uniform distribution of these groups is preferred as patchiness, or (areal) density fluctuations, can enhance attractive correlation effects. ${ }^{41,51-54}$ The precise numbers of 

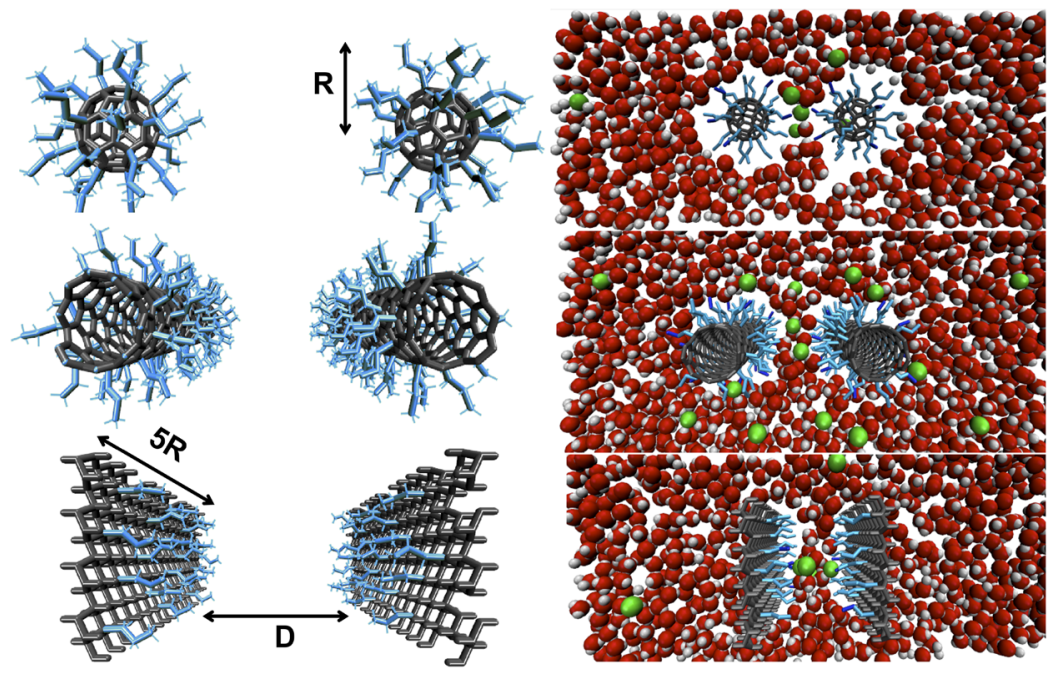

FIG. 1. Left: A sketch of functionalized carbon nanoparticles. The particle backbones and the propyl chains functionalities are shown in grey and blue colors, respectively. CNTs and platelets are held in the parallel orientation. $\sigma=2 R$ is the excluded volume diameter of the functionalized fullerenes, and CNTs defined as the biggest centerof-mass separation at which the propyl end groups on the adjacent particles can be brought into contact. The lengths of the CNT and graphane are 2.5 times the diameter, $\sigma$, of the functionalized fullerene or CNT. $D=r-\sigma$ measures the solvent-accessible space between the functionalized surfaces, where $r$ is the center-of-mass distance. $\sigma=17.3 \AA$ for the functionalized fullerenes and CNTs and $10.5 \AA$ for the platelets. Right: Snapshots from simulated systems with $\sim 25 \%$ of propyl surface groups replaced by ethyl- $\mathrm{NH}_{3}^{+} \mathrm{Cl}^{-}$ones. $\mathrm{Cl}^{-}$counterions are shown in green color. hydrophobic (alkyl) and ionic end groups are listed in Table V of the supplementary material. The inter- and intra-molecular potentials of the solute particles are described using the All Atom Optimized Potentials for Liquid Simulations (OPLSAA) force field ${ }^{55}$ and we use the extended simple point charge model (SPC/ $\left.\mathrm{E}^{56}\right)$ for water. The Lorentz-Berthelot rule is used for mixed Lennard-Jones interactions. Further details of the models with complete sets of force field parameters are provided in Tables I-IV of the supplementary material. In principle, the different curvatures of nanoparticles can affect the partial charges on the particle atoms. These second-order effects are neglected in our simplified approach ${ }^{55}$ as the backbone atoms are buried under the propyl coating and are relatively insignificant compared to the strong influence of ion-bearing functional groups. Our earlier study also revealed a very minor role of $\left(\right.$ small $\left.^{57}\right)$ partial charges on carbon and hydrogen atoms in pristine graphane on its wetting behavior in water. ${ }^{45}$

\section{METHODS}

\section{A. Molecular dynamics/umbrella sampling simulations}

Constant number of molecules $(N)$, pressure $(P)$, and temperature $(T)$ simulations $(N P T)$ of ionic functionalized graphitic nanoparticle systems were performed using the LAMMPS package. ${ }^{58}$ The velocity Verlet algorithm was used to integrate the equations of motion. The SHAKE algorithm was applied to constrain the bonds in water molecules. The simulation time step was 1 fs. The cutoff distance of nonbonded interactions was $12 \AA$. Coulombic forces were handled by the Ewald summation. ${ }^{59}$ The potential of mean force $W(D)$, defined as the free energy at the specified separation $D=r-\sigma$ between the nanoparticles, was calculated using the umbrella sampling (US) method. ${ }^{59}$ A pair of solutes placed in the simulation box with up to $6 \times 10^{3}$ water molecules were initially placed at the separation of $\sim 30-32 \AA$, equilibrated for 9-10 ns and then gradually pulled toward each other using distance decrements of $0.1 \AA ̊ 2100-200$ ps. Selected configurations from this trajectory were used to initiate $2-4 \mathrm{~ns}$ equilibration and up to $6 \mathrm{~ns}$ production runs in a set of predetermined separations to extract the average interparticle force as a function of $D$. While several alternative methods for the calculation of the potential of mean force exist and are equally valid, ${ }^{12,32,35,48,60-62}$ we obtained $W(D)$ by integration of the US mean force (see Ref. 63) from separations at which the simulated forces became statistically indistinguishable from zero. The procedure we described in detail in the previous work ${ }^{42}$ has also been validated by reproducing the $W(D)$ profiles between fullerene particles from Ref. 32. The simulation setups are shown in Fig. 1 right. The details about the simulation runs for all the systems are provided in Table VI of the supplementary material.

\section{RESULTS AND DISCUSSION}

\section{A. Purely hydrophobic particles}

Figure 2 compares the potential of mean force profiles $W(D)$ between a pair of propylated graphane, CNT, or fullerene $\left(\mathrm{C}_{60}\right)$ particles. For easier comparison between the solutes with different sizes, we normalize $W(D)$ by the number of exposed propyl chains facing the opposite particle, which entails division by 19 in the case of graphane, 30 for CNTs, and 12 for fullerenes. Because of their curvatures, however, only a fraction of chains on CNTs, and even fewer in the case of fullerenes, can actually be brought into contact upon approach between the particles. The normalized potential of mean force, $W(D)^{*}$, comprising the direct van der Waals and solvent-induced attractive terms, is therefore the strongest in the planar geometry and the weakest in the spherical geometry. In addition to the well-depth dependence on the curvature, we also observe a shift in the position of the attractive minimum between the fullerenes, compared to the other two structures. With the plates and CNTs, the surface-surface separation ${ }^{54} D=r-\sigma$ at the attractive well is just slightly above 0 , meaning that $r$ barely exceeds the contact distance, $\sigma$, of the given species. The small area of contact between the fullerenes, on the other hand, allows for partial compression or interpenetration of the chains under the effect of van der Waals attraction, shifting the position of the minimum to negative $D$ of $\sim-1.8 \AA$ or about $1 / 2$ the diameter of the terminal methyl groups on propyl chains. As we have shown elsewhere, ${ }^{42}$ the increase in the curvature generally leads to the weakening of both, the direct 


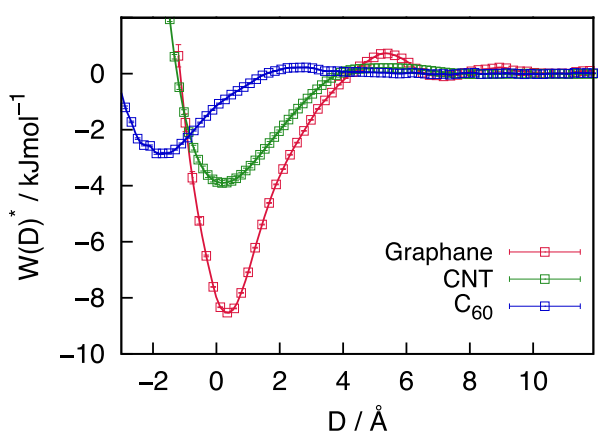

FIG. 2. Normalized potentials of mean force as functions of separation $D$ between a pair of propyl-coated platelets of graphane, CNTs, or fullerenes. $D$ $=r-\sigma$ measures the solvent-accessible spacing between the functionalized surfaces, where $r$ is the center-of-mass distance between the particles. $W(D)^{*}$ between the particles is normalized by the number of chains on the opposing surfaces of the particles: 19 for graphane, 30 for CNT, and 12 for fullerene.

and the solvent mediated attraction, between hydrophobic particles.

\section{B. Particles covered by a mixture of hydrophobic and ionic functionalities}

As shown in Figs. 3 and 4, when the particles acquire a sufficient number of ionic groups, the overall attraction progressively turns to repulsion due to the combined effect of repelling particle charges and increasingly repulsive hydration force. To identify the crossover conditions for different particle curvatures, we gradually modify the surface hydrophobicity of the solutes by replacing the terminal methyl group on the propyl chains with the ammonium chloride or potassium carboxylate groups. We choose target fractions of ionic substitution on the propyl chains $f \approx 0 \%, 12 \%, 25 \%, 50 \%$, $75 \%$, and $100 \%$, rounded to the nearest percentage realizable with an integer number of substituted groups. The actual numbers of ion-substituted groups per particle are listed in Table $\mathrm{V}$ of the supplementary material. We present the $W(D)^{*}$ profiles for all three particle geometries and different extents of ionic substitution in two different ways. In Fig. 3, we compare the results for distinct particle geometries at each of the three preselected degrees of substitution $f \sim 12 \%, 25 \%$, and $100 \%$. Conversely, Fig. 4 compares the $W(D)^{*}$ profiles separately for each geometry at varied extents of ionic functionalization. In both cases, we show the results for cationic $\left(-\mathrm{NH}_{3}^{+} \mathrm{Cl}^{-}\right)$ and anionic $\left(-\mathrm{COO}^{-} \mathrm{K}^{+}\right)$substitutions. The normalization of $W(D)^{*}$ is identical as described in Fig. 2.

While the planar shape of the platelets in the purely hydrophobic form supports stronger attraction than between cylinders or spheres, Figs. 3 and 4 show that the interparticle potential in this geometry is also the most sensitive to the introduction of ionic groups. As the charge density is gradually built up, the repulsion increases most rapidly in the planar geometry. Interpolation between the calculated curves suggests that the repulsion overcomes the attractive terms at $12 \%-16 \%$ ionic substitution $f$ or the average charge density $q_{\mathrm{s}}=f q_{\max }$ between 0.4 and $0.65 e_{\mathrm{o}} \mathrm{nm}^{-2}\left(q_{\max } \sim 4 e_{\mathrm{o}} \mathrm{nm}^{-2}\right.$ is the hypothetical charge density at $100 \%$ ionic functionalization and no counterion binding). For comparison, we observe the crossover to net repulsion between cylindrical particles at $q_{\mathrm{s}}$ between 0.5 and $0.8 e_{\mathrm{o}} \mathrm{nm}^{-2}(14 \% \leq f \leq 20 \%)$ and between 1.2 and $1.6 e_{\mathrm{o}} \mathrm{nm}^{-2}(30 \% \leq f \leq 40 \%)$ with the fullerenes. In all the cases, the lower density corresponds to $-\mathrm{COO}^{-} \mathrm{K}^{+}$ and the higher to $-\mathrm{NH}_{3}^{+} \mathrm{Cl}^{-}$substitutions. The smallest intervention is therefore needed to solubilize the platelet particles that are most prone to association in the nonionic state.

The percentage of ionic substitutions needed to prevent the attraction among the particles increases with the curvature.
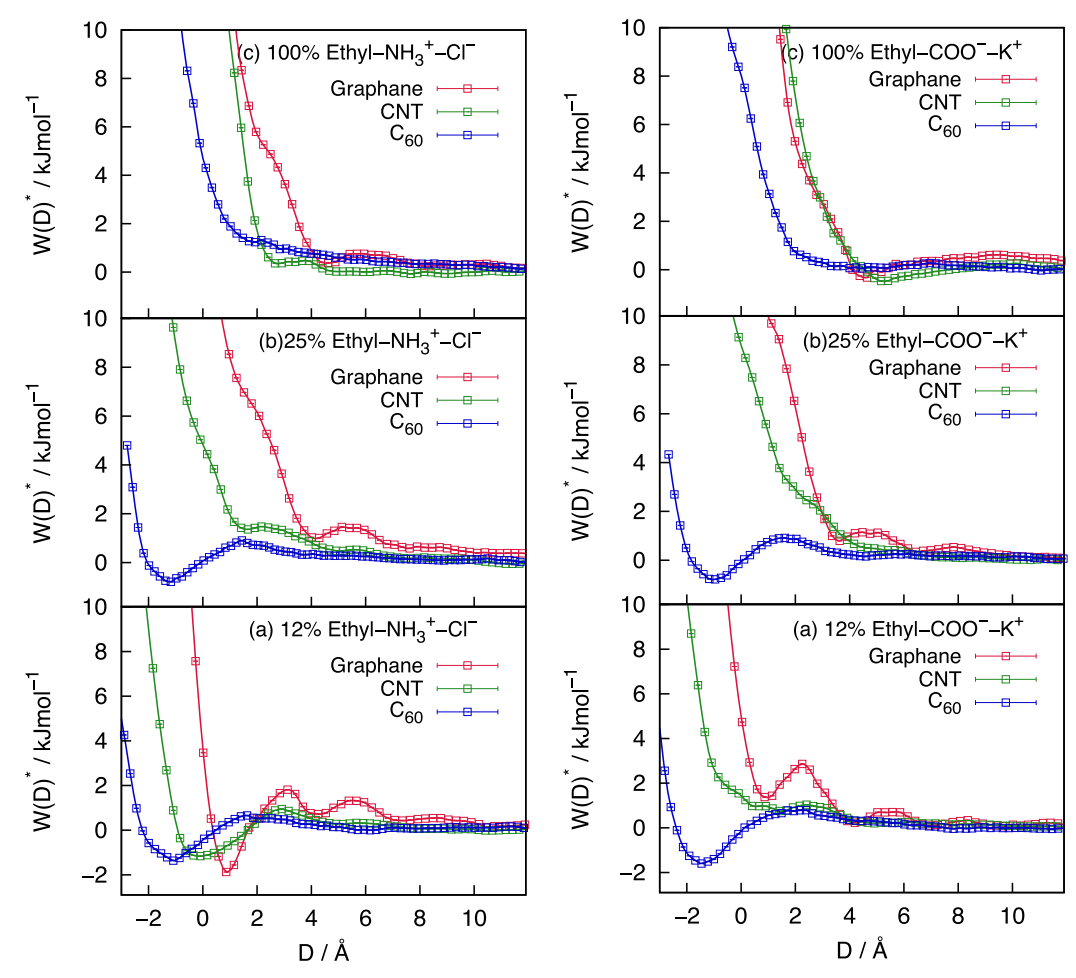

FIG. 3. Variation of the potential of mean force, $W(\mathrm{D})^{*}$ as a function of the interparticle separation $D$ for propylcoated graphitic nanoparticles with varied fractions of propyl groups $f=12 \%$ (a), $25 \%$ (b), or $100 \%$ (c) replaced by ethyl- $\mathrm{NH}_{3}^{+} \mathrm{Cl}^{-}$(left) or ethyl- $\mathrm{COO}^{-} \mathrm{K}^{+}$(right panel) groups. $W(D)^{*}$ is normalized by the number of chains on the opposing particle surfaces (19 for graphane, 30 for CNT, and 12 for fullerene; see Fig. 2). 

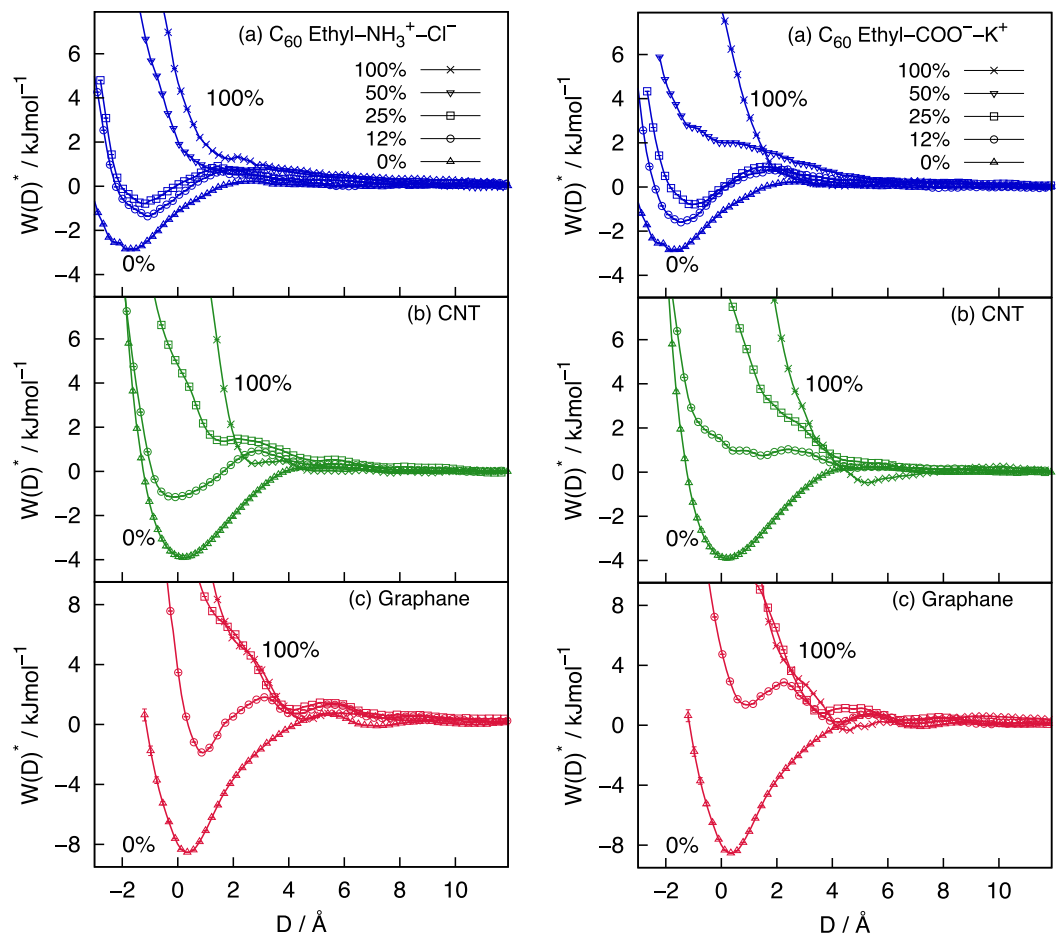

FIG. 4. Variation of the normalized potential of mean force, $W(\mathrm{D})^{*}$ as a function of the interparticle separation $D$ for propyl-coated graphitic nanoparticles with varied fractions of propyl groups $(0 \%-100 \%)$ replaced by ethyl- $\mathrm{NH}_{3}^{+} \mathrm{Cl}^{-}$(left) or ethyl- $\mathrm{COO}^{-} \mathrm{K}^{+}$(right panel) functionalities: functionalized fullerenes (a), carbon nanotubes (b), or graphane (c). $W(D)^{*}$ is normalized by the number of chains on the opposing surfaces of the particles (see the Caption of Fig. 2). The curves corresponding to $50 \%$ substitutions are shown only for the spherical $\left(\mathrm{C}_{60}\right)$ geometry; they are almost identical to the $100 \%$ ones in the cylindrical (CNT) and planar (graphane) geometries.

From our previous work, ${ }^{41}$ we know that a similar dependence does not apply to the charge density needed to bring the contact angle of a hydrocarbon surface below $90^{\circ}$ and to render the water-induced interparticle force repulsive. ${ }^{41}$ The threshold value of the above transition is close to $0.08 e_{\mathrm{o}} \mathrm{nm}^{-241}$ and does not depend significantly on the curvature of the surface. ${ }^{64}$ Changing the sign of the total (as opposed to the solvent-induced part) interaction between adjacent particles from attraction in the absence of charges to overall repulsion in their presence requires an order of magnitude bigger charge densities. The higher charge is needed to offset not only the (weak) solvent-induced term ${ }^{42}$ but also the stronger direct (predominantly van der Waals) attraction between the particles. The main reason for the high effectiveness of functionalization on planar surfaces is that all ionic functionalities on one surface can simultaneously approach the opposite one, producing strong electrostatic repulsion. On the curved particles, a smaller fraction of surface groups can be brought to a comparable proximity of another particle. In addition, the hydration of exposed ionic groups is easier to preserve when these groups are located on curved surfaces of approaching particles than between the planar ones.

\section{Nonspecific saturation effects}

The comparisons between the potential-of-mean-force profiles $W(D)$ at different degrees of ionic functionalization reveal a saturation of the effective particle charge. The saturation is reached at a critical value of $f$; for the planar particles $f_{\text {crit }}$ is below $25 \%$ functionalization. Increasing the fraction of ionic functionalities $f$ beyond $f$ crit produces no significant change in the effective charge and the solvent-averaged interparticle potentials. With cylindrical and spherical solutes, a similar saturation occurs around $f \sim 30 \%$ or $50 \%$, respectively. The trend toward effective-charge ${ }^{65}$ saturation conforms to the concept of ion condensation, ${ }^{66-73}$ which implies electrostatic binding of counterions to offset any excess particle charge above a certain critical value. According to the approximate theory of Manning, ${ }^{67}$ the critical charge density depends on the particle shape and the concentration of shielding ions, $c_{s i}$. For sizeable solutes ( $\sigma$ exceeding the Debye screening length $\kappa^{-1}$ ), the approximate threshold charge densities $q_{\text {crit }}$ in the three principal geometries are ${ }^{67}$

$$
\begin{aligned}
& q_{\text {crit }}^{\text {plane }} \approx-\frac{e_{o} \kappa \ln \left(\kappa l_{B}\right)}{2 \pi l_{B}}, \quad q_{\text {crit }}^{\text {cylinder }}=q_{\text {crit }}^{\text {plane }} \frac{K_{1}(\kappa R)}{K_{0}(\kappa R)}, \\
& q_{\text {crit }}^{\text {sphere }}=q_{\text {crit }}^{\text {plane }}\left(1+\frac{1}{\kappa R}\right),
\end{aligned}
$$

where $R=\sigma / 2, l_{B}=e_{o}^{2} / 4 \pi \varepsilon k_{B} T$ is the Bjerrum length, $\kappa=\left(4 \pi l_{\mathrm{B}} N_{\mathrm{A}} c_{s i}\right)^{1 / 2}, N_{\mathrm{A}}$ is the Avogadro number, $\varepsilon$ is the permittivity of the medium, $k_{\mathrm{B}}$ is the Boltzmann constant, and $K_{\mathrm{O}}$ and $K_{1}$ are the Bessel functions of the 2 nd kind. The possibility of ion condensation exists in all three geometries; however, with spherical particles, it is restricted to finite ion concentrations. ${ }^{67}$ In the strong screening limit, $\kappa \rightarrow \infty$, the interfacial electrostatics becomes insensitive to shape. ${ }^{74}$ The three critical densities converge as the radius of curvature greatly exceeds the screening length $\kappa^{-1}$.

Figure 5 illustrates the predictions from the approximate theory of Manning ${ }^{67}$ for the fractions of nonbonded counterions, $\alpha=\min \left\{1, q_{\text {crit }} / f q_{\max }\right\}$, as functions of the degree of ionic functionalization $f$ for the three principal geometries of the particles. The concentration of free counterions $c_{s i}$ varies in parallel with the product $f \alpha$, spanning the range from 0 to $0.08 \mathrm{~mol} \mathrm{dm}^{-3}$. In a qualitative agreement with the simulation results for ion distributions shown in Fig. 6, the ion binding intensifies in the order from spherical to cylindrical to planar geometry. Theoretical threshold values that determine the effective particle charge in the saturated regime are, however, 


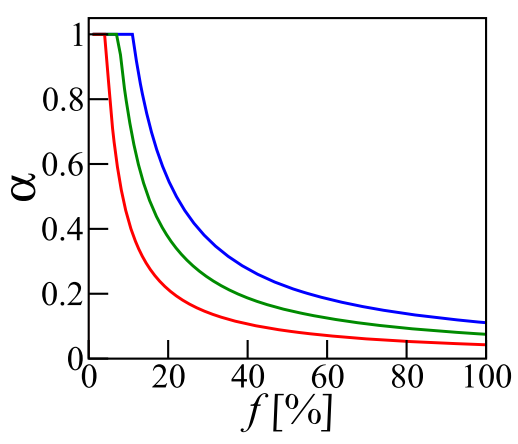

FIG. 5. The fraction of free counterions $\alpha$ as a function of the extent of ionic functionalization $f$ on propylated fullerenes (blue), infinitely long carbon nanotubes (green), or laterally extended graphane platelets (red line) estimated from Manning's theory of ion condensation ${ }^{56}$ for the conditions considered in Figs. 2 and 3. For every particle geometry, there exists a critical surface charge density $q_{c r i t}$, beyond which the effective charge shows saturation with respect to further functionalization. The threshold charge density $q_{c r i t}$ increases from planar to cylindrical to spherical particles. $\alpha$ relates to $q_{\text {crit }}$ as $\alpha=\min \left\{1, q_{\text {crit }} / f\right.$ $\left.q_{\max }\right\}$, where $q_{\max } \sim 4 \mathrm{e}_{\mathrm{o}} / \mathrm{nm}^{2}$ is the surface charge density in a hypothetical state with $f=\alpha=1$.

lower than those observed in the simulations. In addition to the simplifications of the theory, ${ }^{67}$ the difference can be attributed to finite sizes of the simulated particles as opposed to the infinite cylinder length and lateral plate dimensions presumed in the theoretical model.

The restriction on the particle charge density also imposes a limit for the maximal strength of electrostatic repulsion. Once the maximal charge is reached, the repulsion between the particles cannot be strengthened further by additional ionic substitutions. Our simulation results conform to this prediction. Moreover, in several cases, we observe a slight weakening of the repulsion upon increasing the particle charge densities beyond the saturation limit. When this is the case, a shallow attractive minimum can be restored at the interparticle separation corresponding to the increased diameter of the particles that includes the first coordination shell of the counterions. The attractive contribution reflects the correlations between localized but laterally mobile counterions ${ }^{75-87}$ accumulated at the opposing surfaces of a pair of interacting nanoparticles. The change of the counterion-counterion interaction in the locally perturbed solvent environment between the nanoparticles can also play a role. ${ }^{88}$ The correlation effects are stronger with $\mathrm{K}^{+}$counterions than with the $\mathrm{Cl}^{-}$ones because the $\mathrm{Cl}^{-}$ ions tend to penetrate deeper into the nanoparticle coating. For practical purposes, the charge saturation and ion correlation effects establish an optimal degree of ionic functionalization near the condensation limit. A further addition of ionic groups beyond this limit will have no significant effect and can even weaken the interparticle repulsion. In analogy with the (lower) attraction-to-repulsion crossover density, the charge density of maximal repulsion increases with the curvature of the particles.

\section{Ion distribution and specificity}

Figure 6 illustrates the spatial distributions and running coordination numbers of counterions in the ionic atmosphere of charged nanoparticles. The two types of ionic functionalization considered in the present study show distinctly different structural behaviors. The distribution functions of the counterions, $g(D)$, show that the $\mathrm{K}^{+}$ions mostly accumulate next to the nanoparticle (the peak distance $D_{\alpha \beta}$ close to 0 ), with increased tendency to penetrate into the particle coating only at very high degrees of ionization. The $\mathrm{Cl}$-ions, on the other hand, readily penetrate between the coating end groups, with the counterion concentration peaking at the position of ionic $\mathrm{NH}_{3}^{+}$groups at all conditions. The difference reflects contrasting strengths of nonelectrostatic attractive forces between the two types of counterions and the heavy atoms of the functional groups on
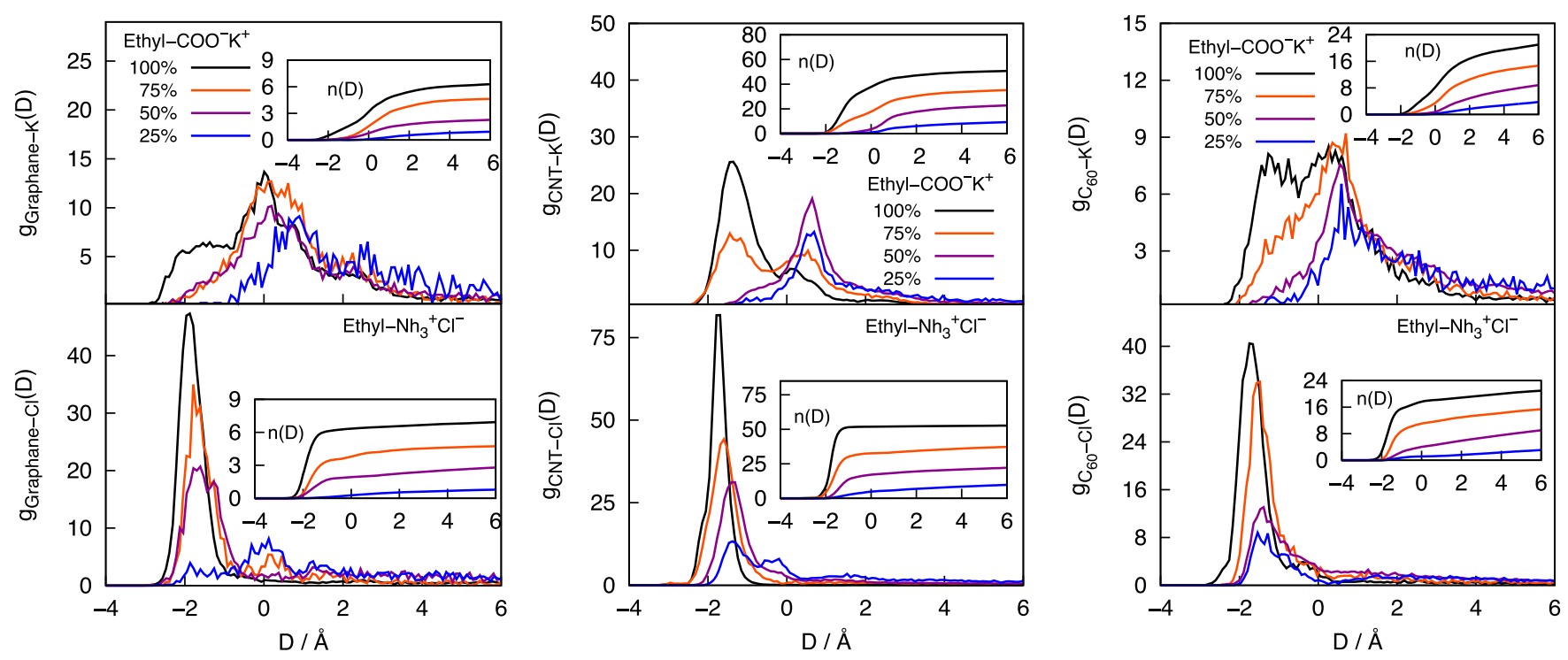

FIG. 6. Radial distribution function between functionalized graphane platelets (left), carbon nanotubes (middle), or fullerenes (right) and $\mathrm{Cl}^{-}$(bottom) or $\mathrm{K}^{+}$ (top) counterions at different percentages of ionic functionalization. $D=D_{\alpha \beta}=r_{\alpha \beta}-\sigma_{\alpha \beta}$, where $r_{\alpha \beta}$ is the center-to-center distance between the two species and $\sigma_{\alpha \beta}=0.5\left(\sigma_{\alpha}+\sigma_{\beta}\right)$ is the contact distance between species $\alpha$ and $\beta$ with diameters $\sigma_{\alpha}$ and $\sigma_{\beta}$. Diameter $\sigma_{\alpha}$ of graphane platelets is $\sim 10.5 \AA$ and $17.3 \AA$ with propylated CNTs and fullerenes, respectively. The diameter of $\mathrm{Cl}^{-}$and $\mathrm{K}^{+}$counterions, $\sigma_{\beta}$, is $4.42 \AA$ and $4.93 \AA$, respectively. The insets show running coordination numbers of counterions $n\left(D_{\alpha \beta}\right)$ as functions of the distance $D_{\alpha \beta}$ from the nanoparticle. 
the particles. The difference is captured through the parameterization of the Lennard-Jones potential parameters of the two ionic species (Table I of the supplementary material) corresponding to different strengths of dispersion interaction due to the very different polarizabilities of the two ionic species. ${ }^{89}$ The nonelectrostatic attraction of the chloride ions to the particle surface translates into stronger shielding of the particle charge, reducing the Coulombic repulsion between the charged nanoparticles. Consistent with this picture, Figs. 3 and 4 show the introduction of a small fraction $f$ of-COOK moieties to be conducive to stronger repulsive effects than with the $-\mathrm{NH}_{3} \mathrm{Cl}$ ones.

The ion-specific binding discussed above also augments the effects of the nonspecific ${ }^{67}$ ion condensation. In all three geometries, the running counterion coordination numbers $n_{\alpha \beta}(D)$,

$$
n_{\alpha \beta}(D)=\rho_{\beta} \int_{o}^{V(D)} g_{\alpha \beta}\left(D_{\alpha \beta}\right) d V,
$$

of the $\mathrm{Cl}^{-}$ions $\left(\beta=\mathrm{Cl}^{-}\right)$reach the plateau value at a few $\AA$ smaller separations than with $\mathrm{K}^{+}$counterions at the identical number density $\rho_{\beta}$ and manifest a smaller slope thereafter. The slope of $n_{\alpha \beta}(D)$ reflects the concentration of free counterions, which also depends on the particle geometry. The slopes of $n_{\alpha \beta}(D)$ at large $D$ stop changing with $f$ above the critical value $f_{\text {crit }}$. The bigger limiting slope $n_{\alpha \beta}(D)$ observed with the fullerenes conforms to the higher fraction of the free counterions in this geometry, in a qualitative agreement with the theoretical predictions. ${ }^{67}$

\section{E. Hydration of charge-bearing nanoparticles}

Pure propyl-coated particles feature strongly hydrophobic hydration that is quite similar in all the three geometries. Figure 7 illustrates water distribution around individual well-separated particles, with the 1st hydration layer comprising water molecules at distances smaller or equal to the distance between the particle and the first minimum of the distribution function $g_{p-O}(D)$. The subscripts $p$ and $O$ denote the nanoparticle and the oxygen atoms of water. When the particles bear no charge, the 1st hydration shell contains between 2 and 2.5 water molecules (depending on the particle shape) per grafted propyl group. In contrast to the hydration of less hydrophobic ${ }^{42,45,46,48,63,90,91}$ pristine CBN particles, here, the 1st density peak $\left(D_{\max }\right)$ is withdrawn $\sim 1 \AA$ away from the direct contact $(D=0)$ with the stretched propyl-coating groups. The tendency toward partial dewetting is characteristic of aqueous interfaces at strongly hydrophobic surfaces, where water attraction to the substrate is much weaker than that among water molecules. ${ }^{92,93}$ Sporadically, a small number of water molecules penetrate between the end groups of the alkyl brush. ${ }^{45}$ With the introduction of even a small fraction of ionic groups, this penetration is greatly enhanced, but the structure changes in a surprising geometry-dependent way. At the interface of functionalized graphane, the original first peak is now attracted into a direct contact with the surface and an even higher one builds up an entire diameter of water inside the platelet's coating. The modified structure is fully developed at only $25 \%$ substitution of methyl end groups by the ionic ones. It is essentially identical with either type of ionic substitution. The surprising independence of water structure on particle ionization and ion type after the rapid initial rearrangement is indicative of a collective penetration process, started by the hydration of charges; however, only a minority of absorbed water molecules can be classified as the hydration water of the ions.

The above changes are not mirrored on the curved surfaces, at which the density of the brush and concomitant steric exclusion increase with the depth. By design, the areal surface densities are equal for all three particle types at the
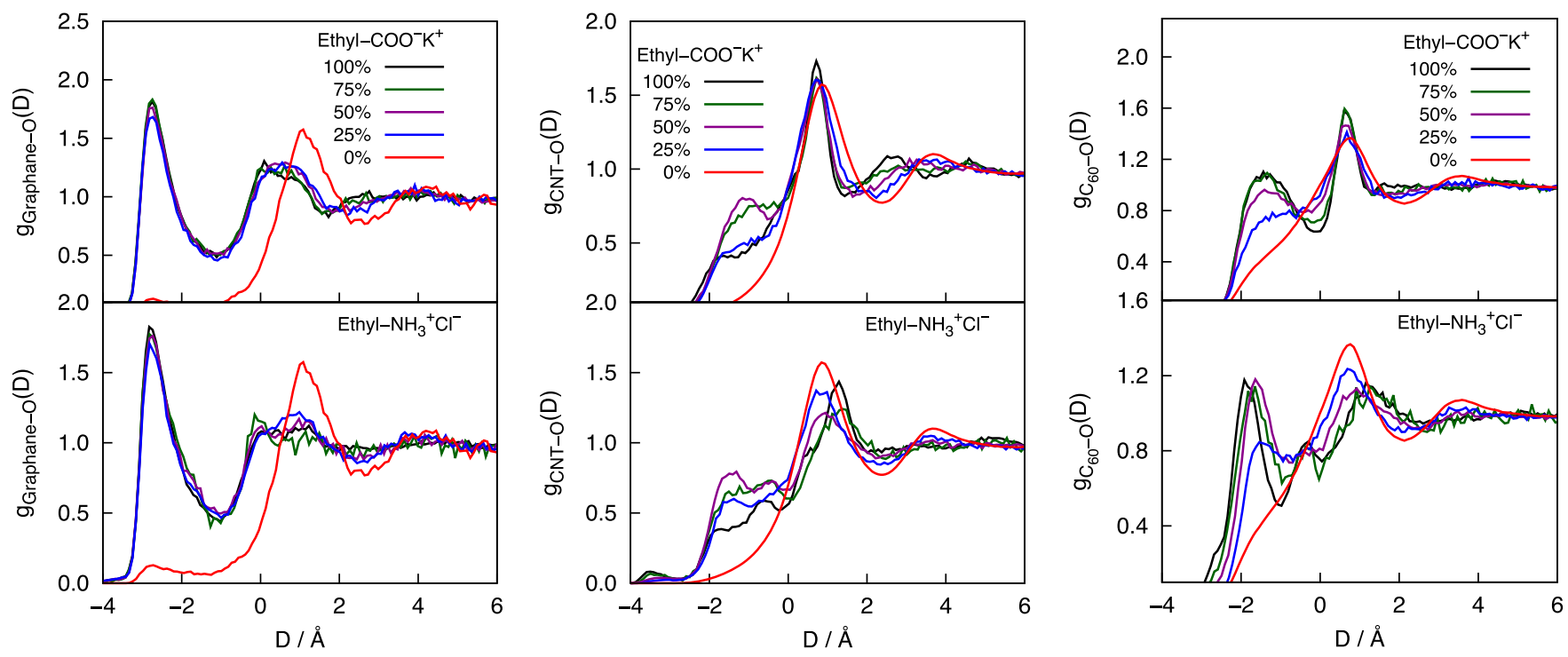

FIG. 7. Radial distribution functions between functionalized graphane platelets (left), carbon nanotubes (middle), or fullerenes (right) and oxygen atoms of water molecules in systems with ethyl- $\mathrm{NH}_{3}^{+} \mathrm{Cl}^{-}$(bottom) or $-\mathrm{COOK}$ moieties (top) at different percentages of ionic functionalization. $D=D_{\alpha \beta}=r_{\alpha \beta}-\sigma_{\alpha \beta}$, where $r_{\alpha \beta}$ is the center to center distance between the two species and $\sigma_{\alpha \beta}=0.5\left(\sigma_{\alpha}+\sigma_{\beta}\right)$ is the contact distance between species $\alpha$ and $\beta$ with diameters $\sigma_{\alpha}$ and $\sigma_{\beta}$. The diameters $\sigma_{\alpha}$ of the particles are equal as in Fig. 5, and the diameter of water, $\sigma_{\beta}$, is $3.166 \AA$. 
particle-solvent contact plane; however, in contrast to the flat surface, the steric hindrance to the uptake of water intensifies with the depth at convex interfaces. The hydration inside the brush on CNTs and fullerenes is therefore less pronounced and peaks at smaller depth, compared to that on a planar solute. The original structural peak slightly away from the particle surface remains dominant, with only a minor shift in position. Because of the steric resistance, the above changes at curved interfaces occur gradually upon increasing particle ionization. This is in contrast to the rapid uptake of water inside the functionality brush of planar particles at a very low density of charged groups, followed by essentially complete saturation beyond $f \sim 25 \%$.

Our results do not indicate any measurable extension of the functionalities in the presence of charges as the propyl chains are essentially stretched even in the neutral state. Nonetheless, the brush hydration also enhances the steric interparticle repulsion at small distances as it hinders the interpenetration of the functionalities belonging to the two approaching surfaces, an effect significant in the fullerene geometry.

Eventual association of the particles results in the expulsion of hydration water from affected particle surfaces. ${ }^{42}$ Because the contact area between associating particles increases from spheres to cylinders to plates and because of the bigger population of water in the brush of planar particles, the hydrophilic hydration of ionized nanoparticles plays a key role in determining the sensitivity of the short-range particle interaction to ionic functionalization (Figs. 3 and 4) in different geometries. Specifically, it supports the easiest charge-induced transition to overall repulsion among the planar particles and the hardest for the spherical ones and envisages reaching saturation with respect to the extent of functionalization in the same order.

While we observe subtle ion-specific effects on the interfacial structure of water, these effects appear to be of secondary importance for the interparticle potentials of mean force in systems with two distinct types of ionic functionalization (Figs. 3 and 4). The nonelectrostatic binding of $\mathrm{Cl}^{-}$ions inside the particle brush, and concomitant increase in ionic shielding compared to the $\mathrm{K}^{+}$system, appears to be the main reason for the stronger effect of the $-\mathrm{COO}^{-} \mathrm{K}^{+}$functionalization on the interparticle repulsion in solution.

\section{CONCLUSIONS}

Ionic functionalization presents an effective means to manipulate the interactions among dispersed nanoparticles in water. Molecular simulations of nanoparticles covered by a mixture of alkyl and ionizing functionalities reveal a strong curvature dependence of the effect of planted charges on the interparticle forces. The sensitivity to the hydrophilic groups, added to solubilize the particles, increases from the spherical particles to the cylindrical and planar ones. While purely hydrophobic platelets are the most prone and the fullerenes the least prone to associate in water, the order is reversed upon introduction of even a small fraction of ionic groups. This comparison is summarized in Fig. 8. The solvent averaged potential between the platelets and nanotubes turns repulsive with around $15 \%$ of alkyl surface groups replaced by

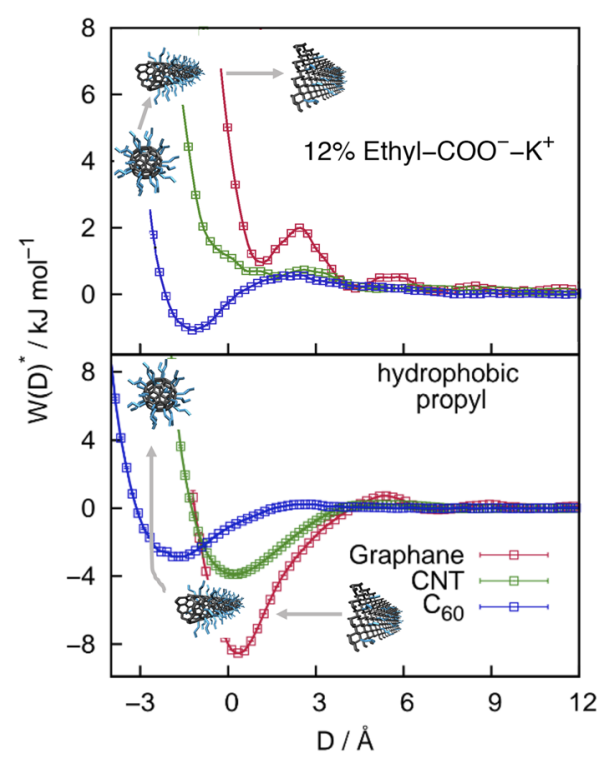

FIG. 8. Bottom: The potential of mean force among propyl-coated graphitic particles shows the weakest attraction in the spherical geometry and the strongest in the planar one. Top: The sensitivity to ionic substitutions increases in the same order. The sequence of profiles $W(D)^{*}$ is already reversed when as few as $12 \%$ of alkyl groups are replaced by ionizing ethyl- $\mathrm{COO}^{-} \mathrm{K}^{+}$ functionalities.

ionic ones. This number is about $1 / 3$ higher for the nanotubes and more than doubled with the fullerenes. The sensitivity to particle shape is attributed to both, the weakening of the Coulombic repulsion with the particle curvature and the increased packing effects that interfere with the hydration of ionic groups inside the coating of convex particles. The effect of functionalization is strongly nonmonotonic, with a saturation limit imposed by counterion condensation. The limiting charge density increases from planar to cylindrical to spherical geometry.

An attractive term due to the correlations among condensed ions can weaken the interparticle repulsion upon the addition of ionic groups in excess of the condensation limit. The optimal areal density of ionic functionalities is the highest for the spherical and the lowest for the platelet particle geometry. These densities can be modulated by ion specific effects due to different strengths of dispersion forces between the counterions and the nanoparticle coating, with the platelets showing the strongest and fullerenes the weakest dependence on the type of the counterion.

\section{SUPPLEMENTARY MATERIAL}

See supplementary material for additional force field information, description of model systems, and details about the simulation runs performed in the potential of mean force calculations.

\section{ACKNOWLEDGMENTS}

We gratefully acknowledge the support of the Office of Basic Energy Sciences, Chemical Sciences, Geosciences, and Biosciences Division of the U.S. Department of Energy (Grant No. DE-SC 0004406). This research used resources of the National Energy Research Scientific Computing 
Center (NERSC), which is supported by the Office of Science of the U.S. Department of Energy under Contract No. DEAC02-05CH11231, and the Extreme Science and Engineering Discovery Environment (XSEDE), which is supported by the National Science Foundation Grant No. OCI-1053575.

${ }^{1}$ Q. Tang, Z. Zhou, and Z. F. Chen, "Graphene-related nanomaterials: Tuning properties by functionalization," Nanoscale 5, 4541 (2013).

${ }^{2}$ J. O. Sofo, A. S. Chaudhari, and G. D. Barber, "Graphane: A twodimensional hydrocarbon," Phys. Rev. B 75, 153401 (2007).

${ }^{3}$ D. C. Elias, R. R. Nair, T. M. G. Mohiuddin, S. V. Morozov, P. Blake, M. P. Halsall, A. C. Ferrari, D. W. Boukhvalov, M. I. Katsnelson, A. K. Geim, and K. S. Novoselov, "Control of graphene's properties by reversible hydrogenation: Evidence for graphane," Science 323, 610 (2009).

${ }^{4}$ M. Terrones, W. K. Hsu, J. P. Hare, H. W. Kroto, H. Terrones, and D. R. M. Walton, "Graphitic structures: From planar to spheres, toroids and helices," Philos. Trans. R. Soc., A 354, 2025 (1996).

${ }^{5}$ Q. Wang, S. H. Zhang, and P. Jena, "Many faces of carbon," Proc. SPIE 10174, 1017405 (2017).

${ }^{6}$ A. K. Geim and I. V. Grigorieva, "Van der Waals heterostructures," Nature 499, 419 (2013).

${ }^{7}$ P. Kumar, S. Karmakar, and H. B. Bohidar, "Anomalous self-aggregation of carbon nanoparticles in polar, nonpolar, and binary solvents," J. Phys. Chem. C 112, 15113 (2008).

${ }^{8}$ K. Scida, P. W. Stege, G. Haby, G. A. Messina, and C. D. Garcia, "Recent applications of carbon-based nanomaterials in analytical chemistry: Critical review," Anal. Chim. Acta 691, 6 (2011).

${ }^{9}$ H. Kim, D. Bedrov, and G. D. Smith, "Molecular dynamics simulation study of the influence of cluster geometry on formation of $\mathrm{C}_{60}$ fullerene clusters in aqueous solution," J. Chem. Theory Comput. 4, 335 (2008).

${ }^{10} \mathrm{~J}$. Brant, H. Lecoanet, and M. R. Wiesner, "Aggregation and deposition characteristics of fullerene nanoparticles in aqueous systems," J. Nanopart. Res. 7, 545 (2005).

${ }^{11}$ M. Alfe, R. Barbella, A. Bruno, P. Minutolo, and A. Ciajolo, "Solution behaviour of $\mathrm{C}_{60}$ fullerene in $\mathrm{N}$-methylpyrrolidinone/toluene mixtures," Carbon 43, 665 (2005).

${ }^{12}$ S. M. Mortuza and S. Banerjee, "Molecular modeling study of agglomeration of [6,6]-phenyl-C61-butyric acid methyl ester in solvents," J. Chem. Phys. 137, 244308 (2012).

${ }^{13}$ D. Jariwala, V. K. Sangwan, L. J. Lauhon, T. J. Marks, and M. C. Hersam, "Carbon nanomaterials for electronics, optoelectronics, photovoltaics, and sensing," Chem. Soc. Rev. 42, 2824 (2013).

${ }^{14}$ M. V. Khodakovskaya, K. de Silva, D. A. Nedosekin, E. Dervishi, A. S. Biris, E. V. Shashkov, E. I. Galanzha, and V. P. Zharov, "Complex genetic, photothermal, and photoacoustic analysis of nanoparticle-plant interactions," Proc. Natl. Acad. Sci. U. S. A. 108, 1028 (2011).

${ }^{15}$ M. Mahmood, D. A. Casciano, T. Mocan, C. Iancu, Y. Xu, L. Mocan, D. T. Iancu, E. Dervishi, Z. R. Li, M. Abdalmuhsen, A. R. Biris, N. Ali, P. Howard, and A. S. Biris, "Cytotoxicity and biological effects of functional nanomaterials delivered to various cell lines," J. Appl. Toxicol. 30, 74 (2010).

${ }^{16}$ Y. B. Zhang, S. F. Ali, E. Dervishi, Y. Xu, Z. R. Li, D. Casciano, and A. S. Biris, "Cytotoxicity effects of graphene and single-wall carbon nanotubes in neural phaeochromocytoma-derived pc12 cells," ACS Nano 4, 3181 (2010).

${ }^{17}$ Z. Liu, S. Tabakman, S. Sherlock, X. L. Li, Z. Chen, K. L. Jiang, S. S. Fan, and H. J. Dai, "Multiplexed five-color molecular imaging of cancer cells and tumor tissues with carbon nanotube Raman tags in the near-infrared," Nano Res. 3, 222 (2010).

${ }^{18}$ S. Foley, C. Crowley, M. Smaihi, C. Bonfils, B. F. Erlanger, P. Seta, and C. Larroque, "Cellular localisation of a water-soluble fullerene derivative," Biochem. Biophys. Res. Commun. 294, 116 (2002).

${ }^{19}$ Z. Liu, M. Winters, M. Holodniy, and H. J. Dai, "siRNA delivery into human $\mathrm{T}$ cells and primary cells with carbon-nanotube transporters," Angew. Chem., Int. Ed. 46, 2023 (2007)

${ }^{20}$ S. Prylutska, R. Bilyy, M. Overchuk, A. Bychko, K. Andreichenko, R. Stoika, V. Rybalchenko, Y. Prylutskyy, N. G. Tsierkezos, and U. Ritter, "Water-soluble pristine fullerenes $\mathrm{C}_{60}$ increase the specific conductivity and capacity of lipid model membrane and form the channels in cellular plasma membrane," J. Biomed. Nanotechnol. 8, 522 (2012).

${ }^{21}$ V. K. Periya, I. Koike, Y. Kitamura, S. I. Iwamatsu, and S. Murata, "Hydrophilic [60]fullerene carboxylic acid derivatives retaining the original 60 pi electronic system," Tetrahedron Lett. 45, 8311 (2004).
${ }^{22}$ J. Cerar and J. Skerjanc, "Water-soluble fullerenes. 2. Sodium fullerenehexamalonate $T_{h}-\mathrm{C}_{66}(\mathrm{COONa})_{12}$, a highly asymmetric electrolyte," J. Phys. Chem. B 104, 727 (2000).

${ }^{23}$ Z. Wang, Z. Lu, Y. Zhao, and X. Gao, "Oxidation-induced watersolubilization and chemical functionalization of fullerenes $\mathrm{C}_{60}$, $\mathrm{gd} @ \mathrm{C}_{60}$ and gd@ $\mathrm{C}_{82}$ : Atomistic insights into the formation mechanisms and structures of fullerenols synthesized by different methods," Nanoscale 7, 2914 (2015).

${ }^{24}$ K. S. Subrahmanyam, S. R. C. Vivekchand, A. Govindaraj, and C. N. R. Rao, "A study of graphenes prepared by different methods: Characterization, properties and solubilization," J. Mater. Chem. 18, 1517 (2008).

${ }^{25}$ D. Tasis, K. Papagelis, M. Prato, I. Kallitsis, and C. Galiotis, "Watersoluble carbon nanotubes by redox radical polymerization," Macromol. Rapid Commun. 28, 1553 (2007).

${ }^{26}$ Y. Si and E. T. Samulski, "Synthesis of water soluble graphene," Nano Lett. 8, 1679 (2008).

${ }^{27}$ K. A. Worsley, P. Ramesh, S. K. Mandal, S. Niyogi, M. E. Itkis, and R. C. Haddon, "Soluble graphene derived from graphite fluoride," Chem. Phys. Lett. 445, 51 (2007)

${ }^{28}$ S. W. Lee, B. S. Kim, S. Chen, Y. Shao-Horn, and P. T. Hammond, "Layerby-layer assembly of all carbon nanotube ultrathin films for electrochemical applications," J. Am. Chem. Soc. 131, 671 (2009).

${ }^{29}$ D. G. Deryabin, O. K. Davydova, Z. Z. H. Yankina, A. S. Vasilchenko, S. A. Miroshnikov, A. B. Kornev, A. V. Ivanchikhina, and P. A. Troshin, "The activity of [60]fullerene derivatives bearing amine and carboxylic solubilizing groups against Escherichia coli: A comparative study," J. Nanomater. 2014, 907435 .

${ }^{30}$ N. Tsao, P. P. Kanakamma, T. Y. Luh, C. K. Chou, and H. Y. Lei, "Inhibition of Escherichia coli-induced meningitis by carboxyfullerence," Antimicrob. Agents Chemother. 43, 2273 (1999).

${ }^{31}$ N. Jha, P. Ramesh, E. Bekyarova, X. J. Tian, F. H. Wang, M. E. Itkis, and R. C. Haddon, "Functionalized single-walled carbon nanotube-based fuel cell benchmarked against US DOE 2017 technical targets," Sci. Rep. 3, 2257 (2013).

${ }^{32} \mathrm{~S}$. Banerjee, "Molecular dynamics study of self-agglomeration of charged fullerenes in solvents," J. Chem. Phys. 138, 044318 (2013).

${ }^{33}$ A. R. A. Dezfoli, M. A. Mehrabian, and H. H. Rafsanjani, "Structural properties of water around uncharged and charged carbon nanotubes," Korean J. Chem. Eng. 30, 693 (2013).

${ }^{34}$ C. Eun and M. L. Berkowitz, "Origin of the hydration force: Water-mediated interaction between two hydrophilic plates," J. Phys. Chem. B 113, 13222 (2009).

${ }^{35} \mathrm{~L}$. Lu and M. L. Berkowitz, "Hydration force between model hydrophilic surfaces: Computer simulations," J. Chem. Phys. 124, 101101 (2006).

${ }^{36} \mathrm{~L}$. Lu and M. L. Berkowitz, "The effect of water structure and surface charge correlations on the hydration force acting between model hydrophilic surfaces," Mol. Phys. 104, 3607 (2006).

${ }^{37}$ C. Eun and M. L. Berkowitz, "Molecular dynamics simulation study of the water-mediated interaction between zwitterionic and charged surfaces," J. Chem. Phys. 136, 024501 (2012).

${ }^{38}$ J. Y. Li, T. Liu, X. Li, L. Ye, H. J. Chen, H. P. Fang, Z. H. Wu, and R. H. Zhou, "Hydration and dewetting near graphite- $\mathrm{CH}_{3}$ and graphite- $\mathrm{COOH}$ plates," J. Phys. Chem. B 109, 13639 (2005).

${ }^{39}$ J. Wang, D. Bratko, and A. Luzar, "Probing surface tension additivity on chemically heterogeneous surfaces: A molecular approach," Proc. Natl. Acad. Sci. U. S. A. 108, 6374 (2011).

${ }^{40} \mathrm{P}$. H. Shah and R. C. Batra, "Effect of covalent functionalization on Young's modulus of a single-wall carbon nanotube," in Modeling of Carbon Nanotubes, Graphene and Their Composites, edited by K. I. Tserpes and N. Silvestre (Springer, 2014), Vol. 188, p. 111.

${ }^{41}$ D. Vanzo, D. Bratko, and A. Luzar, "Tunable wetting of surfaces with ionic functionalities," J. Phys. Chem. C 116, 15467 (2012).

${ }^{42}$ B. S. Jabes, D. Bratko, and A. Luzar, "Universal repulsive contribution to the solvent-induced interaction between sizable, curved hydrophobes," J. Phys. Chem. Lett. 7, 3158 (2016).

${ }^{43}$ H. Acharya, S. Ranganathan, S. N. Jamadagni, and S. Garde, "Mapping hydrophobicity at the nanoscale: Applications to heterogeneous surfaces and proteins," Faraday Discuss. 146, 353 (2010).

${ }^{44}$ R. Evans and M. C. Stewart, "The local compressibility of liquids near nonadsorbing substrates: A useful measure of solvophobicity and hydrophobicity?," J. Phys.: Condens. Matter 27, 194111 (2015).

${ }^{45}$ D. Vanzo, D. Bratko, and A. Luzar, "Wettability of pristine and alkylfunctionalized graphane," J. Chem. Phys. 137, 034707 (2012). 
${ }^{46}$ L. W. Li, D. Bedrov, and G. D. Smith, "Water-induced interactions between carbon nanoparticles," J. Phys. Chem. B 110, 10509 (2006).

${ }^{47}$ C. Gu, C. Ray, S. Guo, and B. B. Akhremitchev, "Single-molecule force spectroscopy measurements of interactions between $\mathrm{C}_{60}$ fullerene molecules," J. Phys. Chem. C 111, 12898 (2007).

${ }^{48}$ L. W. Li, D. Bedrov, and G. D. Smith, "A molecular-dynamics simulation study of solvent-induced repulsion between $\mathrm{C}_{60}$ fullerenes in water," J. Chem. Phys. 123, 204504 (2005).

${ }^{49}$ C. D. Bain, J. Evall, and G. M. Whitesides, "Formation of monolayers by the coadsorption of thiols on gold: Variation in the head group, tail group, and solvent," J. Am. Chem. Soc. 111, 7155 (1989).

${ }^{50}$ Y. Arima and H. Iwata, "Effect of wettability and surface functional groups on protein adsorption and cell adhesion using well-defined mixed self-assembled monolayers," Biomaterials 28, 3074 (2007).

${ }^{51}$ D. Bratko and A. K. Chakraborty, "Ion-ion correlations in quenched disordered media," J. Chem. Phys. 104, 7700 (1996).

${ }^{52}$ A. K. Chakraborty, D. Bratko, and D. Chandler, "Diffusion of ionic penetrants in charged disordered media," J. Chem. Phys. 100, 1528 (1994).

${ }^{53}$ D. Bratko, A. K. Chakraborty, and E. I. Shakhnovich, "Recognition between random heteropolymers and multifunctional disordered surfaces," Chem. Phys. Lett. 280, 46 (1997).

${ }^{54}$ S. D. Overduin and G. N. Patey, "Solvent phase behavior and the interaction of uniform and patterned solutes," J. Chem. Phys. 123, 194505 (2005).

${ }^{55}$ W. L. Jorgensen, D. S. Maxwell, and J. Tirado-Rives, "Development and testing of the OPLS all-atom force field on conformational energetics and properties of organic liquids," J. Am. Chem. Soc. 118, 11225 (1996).

${ }^{56}$ H. J. C. Berendsen, J. R. Grigera, and T. P. Straatsma, "The missing term in effective pair potentials,” J. Phys. Chem. 91, 6269 (1987).

${ }^{57}$ S. Lebegue, M. Klintenberg, O. Eriksson, and M. I. Katsnelson, "Accurate electronic band gap of pure and functionalized graphane from GW calculations," Phys. Rev. B 79, 245117 (2009).

${ }^{58}$ S. Plimpton, "Fast parallel algorithms for short-range molecular-dynamics," J. Comput. Phys. 117, 1 (1995).

${ }^{59}$ D. Frenkel and B. Smit, Understanding Molecular Simulation, from Algorithms to Applications (Academic Press, San Diego, 2002).

${ }^{60}$ E. Darve and A. Pohorille, "Calculating free energies using average force," J. Chem. Phys. 115, 9169 (2001).

${ }^{61}$ E. Darve, M. A. Wilson, and A. Pohorille, "Calculating free energies using a scaled-force molecular dynamics algorithm," Mol. Simul. 28, 113 (2002).

${ }^{62}$ N. Choudhury and B. M. Pettitt, "On the mechanism of hydrophobic association of nanoscopic solutes,” J. Am. Chem. Soc. 127, 3556 (2005).

${ }^{63}$ R. Zangi, "Are buckyballs hydrophobic?," J. Phys. Chem. B 118, 12263 (2014).

${ }^{64}$ J. Wang, D. Bratko, and A. Luzar, "Length-scale dependence of hydration free energy: Effect of solute charge,” J. Stat. Phys. 145, 253 (2011).

${ }^{65}$ R. Kjellander, "Fundamental aspects of electrostatic interactions and charge renormalization in electrolyte systems," Colloid J. 69, 20 (2007).

${ }^{66} \mathrm{G}$. S. Manning, "Molecular theory of polyelectrolyte solutions with implications to electrostatic properties of polynucleotides," Q. Rev. Biophys. 11, 179 (1978)

${ }^{67}$ G. S. Manning, "Counterion condensation on charged spheres, cylinders, and planes," J. Phys. Chem. B 111, 8554 (2007).

${ }^{68}$ G. S. Manning, "The interaction between a charged wall and its counterions: A condensation theory," J. Phys. Chem. B 114, 5435 (2010).

${ }^{69} \mathrm{~S}$. Engstrom and H. Wennerstrom, "Ion condensation on planar surfacesSolution of Poisson-Boltzmann equation for two parallel charged plates," J. Phys. Chem. 82, 2711 (1978).

${ }^{70}$ G. V. Ramanathan, "Statistical-mechanics of electrolytes and polyelectrolytes. III. The cylindrical Poisson-Boltzmann equation," J. Chem. Phys. 78, 3223 (1983).
${ }^{71}$ I. Rouzina and V. A. Bloomfield, "Macroion attraction due to electrostatic correlation between screening counterions. 1. Mobile surface-adsorbed ions and diffuse ion cloud," J. Phys. Chem. 100, 9977 (1996).

${ }^{72}$ P. Linse, "Structure, phase stability, and thermodynamics in charged colloidal solutions," J. Chem. Phys. 113, 4359 (2000).

${ }^{73}$ R. R. Netz and H. Orland, "Variational charge renormalization in charged systems,” Eur. Phys. J. E 11, 301 (2003).

${ }^{74}$ R. A. Curtis and L. Lue, "Electrolytes at spherical dielectric interfaces," J. Chem. Phys. 123, 174702 (2005).

${ }^{75}$ R. Kjellander and S. Marcelja, "Inhomogeneous Coulomb fluids with image interactions between planar surfaces. I," J. Chem. Phys. 82, 2122 (1985).

${ }^{76}$ L. Guldbrand, B. Jonsson, H. Wennerstrom, and P. Linse, "Electrical doublelayer forces-A Monte-Carlo study," J. Chem. Phys. 80, 2221 (1984).

${ }^{77}$ D. Bratko, B. Jonsson, and H. Wennerstrom, "Electrical double-layer interactions with image charges," Chem. Phys. Lett. 128, 449 (1986).

${ }^{78}$ L. Guldbrand, L. G. Nilsson, and L. Nordenskiold, "A Monte-Carlo simulation study of electrostatic forces between hexagonally packed DNA double helices," J. Chem. Phys. 85, 6686 (1986).

${ }^{79}$ C. E. Woodward, B. Jonsson, and T. Akesson, "The ionic correlation contribution to the free-energy of spherical double-layers," J. Chem. Phys. 89, 5145 (1988)

${ }^{80}$ A. Luzar and D. Bratko, "Electric double-layer interactions in reverse micellar systems_A Monte-Carlo simulation study," J. Chem. Phys. 92, 642 (1990).

${ }^{81}$ D. Bratko, C. E. Woodward, and A. Luzar, "Charge fluctuation in reverse micelles,” J. Chem. Phys. 95, 5318 (1991).

${ }^{82}$ B. Hribar and V. Vlachy, "Evidence of electrostatic attraction between equally charged macroions induced by divalent counterions," J. Phys. Chem. B 101, 3457 (1997)

${ }^{83}$ R. J. Mashl and N. Gronbech-Jensen, "Effective interactions between rigid polyelectrolytes and like-charged planar surfaces," J. Chem. Phys. 109, 4617 (1998).

${ }^{84}$ J. Z. Wu, D. Bratko, and J. M. Prausnitz, "Interaction between like-charged colloidal spheres in electrolyte solutions," Proc. Natl. Acad. Sci. U. S. A. 95, 15169 (1998)

${ }^{85}$ M. Lund and B. Jonsson, "Driving forces behind ion-ion correlations," J. Chem. Phys. 125, 236101 (2006)

${ }^{86}$ M. Lund and B. Jonsson, "Charge regulation in biomolecular solution," Q. Rev. Biophys. 46, 265 (2013).

${ }^{87}$ G. N. Patey, "The interaction of two spherical colloidal particles in electrolyte solution. An application of the hypernetted-chain approximation," J. Chem. Phys. 72, 5763 (1980).

${ }^{88}$ F. Otto and G. N. Patey, "Forces between like-charged walls in electrolyte solution: Molecular solvent effects at the McMillan-Mayer level,”J. Chem. Phys. 112, 8939 (2000).

${ }^{89}$ F. W. Tavares, D. Bratko, H. W. Blanch, and J. M. Prausnitz, "Ion-specific effects in the colloid-colloid or protein-protein potential of mean force: Role of salt-macroion van der Waals interactions," J. Phys. Chem. B 108, 9228 (2004)

${ }^{90}$ N. Choudhury, "A molecular dynamics simulation study of buckyballs in water: Atomistic versus coarse-grained models of $\mathrm{C}_{60}$," J. Chem. Phys. 125, 034502 (2006)

${ }^{91}$ N. Choudhury, "Dynamics of water in the hydration shells of $\mathrm{C}_{60}$ : Molecular dynamics simulation using a coarse-grained model," J. Phys. Chem. B 111, 10474 (2007).

${ }^{92}$ D. Bratko, R. A. Curtis, H. W. Blanch, and J. M. Prausnitz, "Interaction between hydrophobic surfaces with metastable intervening liquid," J. Chem. Phys. 115, 3873 (2001).

${ }^{93}$ D. M. Huang and D. Chandler, "The hydrophobic effect and the influence of solute-solvent attractions,” J. Phys. Chem. B 106, 2047 (2002). 